\title{
Age-related differences in neuroinflammatory responses associated with a distinct profile of regulatory markers on neonatal microglia
}

\author{
Leah B Christensen, Tyson A Woods, Aaron B Carmody, Byron Caughey and Karin E Peterson*
}

\begin{abstract}
Background: The perinatal period is one in which the mammalian brain is particularly vulnerable to immune-mediated damage. Early inflammation in the central nervous system (CNS) is linked with long-term impairment in learning and behavior, necessitating a better understanding of mediators of neuroinflammation. We therefore directly examined how age affected neuroinflammatory responses to pathogenic stimuli.

Methods: In mice, susceptibility to neurological damage changes dramatically during the first few weeks of life. Accordingly, we compared neuroinflammatory responses to pathogen associated molecular patterns (PAMPs) of neonatal (two day-old) and weanling (21 day-old) mice. Mice were inoculated intracerebrally with PAMPs and the cellular and molecular changes in the neuroinflammatory response were examined.

Results: Of the 12 cytokines detected in the CNS following toll-like receptor 4 (TLR4) stimulation, ten were significantly higher in neonates compared with weanling mice. A similar pattern of increased cytokines in neonates was also observed with TLR9 stimulation. Analysis of cellular responses indicated a difference in microglial activation markers in the CNS of neonatal mice and increased expression of proteins known to modulate cellular activation including CD11a, F4/80 and CD172a. We also identified a new marker on microglia, SLAMF7, which was expressed at higher levels in neonates compared with weanlings.

Conclusions: A unique neuroinflammatory profile, including higher expression of several proinflammatory cytokines and differential expression of microglial markers, was observed in brain tissue from neonates following TLR stimulation. This increased neuroinflammatory response to PAMPs may explain why the developing brain is particularly sensitive to infection and why infection or stress during this time can lead to long-term damage in the CNS.
\end{abstract}

\section{Background}

During the perinatal period, the mammalian brain is developing rapidly and is particularly sensitive to inflammation or maternal stress. Gestational viral, bacterial and parasitic infections have been linked to neurological illnesses in offspring, including cerebral palsy and schizophrenia [1-3]. Additionally, perinatal infection is a factor for developing neurodegenerative diseases such as Alzheimer's and Parkinson's later in life [4-6]. Current research suggests that it may not be the infectious agent per se but the immune response that is causing the neurological damage $[2,7,8]$. One useful model is the

\footnotetext{
* Correspondence: petersonka@niaid.nih.gov

Laboratory of Persistent Viral Diseases, Rocky Mountain Laboratories, National Institute of Allergy and Infectious Diseases, National Institutes of Health, Hamilton MT 59840, USA
}

neonatal mouse, where cortical development roughly corresponds to the human fetus midway through gestation $[9,10]$. Neonatal rodent models have demonstrated that perinatal immune stimulation with either infectious agents or Toll-like receptor (TLR) ligands can produce developmental and behavioral changes similar to those observed in human neurological illnesses, including alterations in learning and memory $[8,11]$. Understanding the immune response in the CNS during the perinatal period is necessary in order to understand how developmental abnormalities and neurological damage may occur.

Studies of immune cells in neonates indicate that the peripheral immune response is often suppressed in response to infection or immune stimuli [12-15]. As such, neonates and infants are more susceptible to viral and 
bacterial infections compared with adults [12,16,17]. This suppression may be due in part to reduced activation of neonatal monocytes to TLR activation compared with adult monocytes [13-15,18,19]. Inability to respond to viral or bacterial infections may allow infections to spread and persist in the neonatal host, resulting in increased damage to healthy tissues compared with adults.

It is unclear whether the suppressed immune response observed in the periphery of neonates during development is also observed in the CNS. During development, microglial cells, which are considered to be the resident macrophage population of the brain, are in an active state [20-22]. Microglia are derived from the yolk sac and migrate into the CNS during the perinatal period where they are actively involved in pruning synapses from neurons [22,23]. Following this process, microglia undergo a ramification process where they become quiescent and persist in this state unless activated by insult or injury [20]. Thus, microglia may actually be more activated, and thus possibly more responsive to immune stimuli, during the perinatal/neonatal period compared with adults.

Here we have examined neuroinflammatory responses in neonatal and weanling mice by inoculating them with two pathogen associated molecular patterns (PAMPs) that have been used for modeling neurodevelopmental illness: lipopolysaccharide (LPS), the ligand for TLR4, and unmethylated CpG oligodeoxynucleotides (CpGODN), the ligand for TLR9. Increased expression of proinflammatory cytokines and chemokines, as well as other neuroinflammatory markers, were significantly elevated in the brains of neonatal mice compared with weanling mice. We then examined the myeloid cell population within the CNS to examine possible mediators of the differential inflammatory response.

\section{Methods}

\section{Ethics statement}

All animal research was carried out in adherence with protocols approved by the National Institutes of Health Rocky Mountain Laboratories Animal Care and Use Committee with animal protocols 2008-46 and 2009-70.

\section{Animal models and inoculation of TLR agonists}

All mice were housed and maintained by the Rocky Mountain Laboratories Veterinary Branch (Hamilton, MT, USA). Mice were maintained under pathogen-free conditions with regular light/dark cycles and given food and water ad libitum. For inoculations, neonatal (two dayold) C57BL/10 mice were anaesthetized by hypothermia, while weanling (21 day-old) mice were anaesthetized by isofluorane inhalation prior to inoculation. Mice were inoculated intracerebrally (ic), using a Hamilton syringe with a 33-gauge needle, once in each hemisphere with a solution volume of $3 \mu \mathrm{l}$ per hemisphere. Mice were inoculated with either LPS, CpG-ODN or as an inoculation control, PBS. We used LPS and CpG-ODN concentrations that, based on preliminary studies, were expected to elicit strong TLR-mediated responses without being lethal. Mice were inoculated with either $0.5 \mu \mathrm{g}$ LPS per gram of body weight or $0.125 \mu \mathrm{g}$ CpG-ODN per gram of body weight. For neonatal mice, this meant a total inoculum of $1 \mu \mathrm{g}$ of LPS or $0.25 \mu \mathrm{g}$ (40 picomoles) of CpG-ODN. For three week old mice, this translated to $3.8 \mu \mathrm{g}$ of LPS or $0.95 \mu \mathrm{g}$ of CpG-ODN. Uninoculated mice were also used as additional controls. To control for any possible variations between litters, agonist preparation or inoculations, all experimental groups contained mice from different litters that were inoculated on different days.

At the indicated time points, all animals were anaesthetized by inhalation of isofluorane and euthanized using axillary incision. Brain tissue was snap frozen in liquid nitrogen and stored at $-80^{\circ} \mathrm{C}$ until use.

\section{TLR agonists}

TLR4 agonist ultra-pure LPS (catalog number tlrl3pelps) and TLR9 agonist phosphorothioated CpG-ODN type B [5' -tcc atg acg ttc ctg acg tt-3'] (catalog number tlrl-1826) were purchased from InvivoGen (San Diego, CA, USA). Agonist stocks were suspended in endotoxinfree water, aliquoted and stored at $-20^{\circ} \mathrm{C}$. Immediately prior to use, agonists were diluted in endotoxin-free, PBS-buffered solution.

\section{Protein quantification}

For protein quantification, brain tissue was weighed and homogenized in Bio-Plex cell lysis buffer (Bio-Rad, Hercules, CA, USA; catalog number 171-304012) containing PMSF (Sigma, St. Louis, MO, USA; catalog number P-7626) and Complete Mini Protease Inhibitor Cocktail (Roche, Basel, CH; catalog number 11836153001) as previously described [24]. All samples were diluted to a final concentration of $300 \mathrm{mg} / \mathrm{ml}$ of brain tissue in lysis buffer. Samples were then centrifuged at $4,500 \times g$ for 15 minutes at $4^{\circ} \mathrm{C}$ to remove debris. Cytokine and chemokine protein levels in brain homogenate supernatants were analyzed using the Invitrogen Mouse Cytokine Twenty-Plex Antibody Bead kit (Carlsbad, CA, USA; catalog number LMC006) on a Bio-Rad Bio-Plex 200 system (Hercules, CA, USA). Individual protein concentrations were calculated using standard curves generated from standards provided with the Twenty-Plex kit.

\section{Quantification of mRNA expression by real-time PCR}

Total RNA was extracted from brain tissue using the Qiagen RNeasy Mini Kit (Valencia, CA, USA; catalog number 74106) per the manufacturer's instructions. RNA was then treated with DNase (Ambion, Foster City, 
CA, USA; catalog number AM2224) for 30 minutes at $37^{\circ} \mathrm{C}$, followed by a final purification and concentration using the Zymo Research RNA Clean-up kit (catalog number R1018). Complimentary DNA (cDNA) was generated from the isolated RNA using the iScript cDNA Synthesis kit (Bio-Rad, Hercules, CA, USA; catalog number 170-8891). All primers were designed using Primer3 and were gene-specific in blast searches performed using the National Center for Biotechnology Information database as previously described [24]. Real-time PCR was performed using iTAQ SYBR Green Supermix with ROX (Bio-Rad, Hercules, CA, USA, catalog number 1725852) on an Applied Biosystems (Grand Island, NY, USA) PRISM 7900HT instrument. All samples were run in triplicate. The baseline was automatically set and the $\mathrm{C}_{\mathrm{T}}$ was manually set to intersect the mid-log phase of PCR curves at 0.19. Dissociation curves were used to verify that only a single gene product was amplified in each sample. Data analysis was completed following the published criteria for real-time quantitative PCR method using the comparative $C_{T}$ method $[25,26]$. Gene expression was calculated relative to the average expression of three housekeeping genes (Actb, Gusb, Rpl32) for each sample within an experiment. The same housekeeping genes were used for each experiment. Data are shown as the percent of the average of the housekeeping genes (\% of housekeeping genes). RNA that was not reverse transcribed and water were used as negative controls.

\section{Preparation of brain tissue for flow cytometry}

Animals were anaesthetized by inhalation of isofluorane, followed by perfusion through the left ventricle of the heart with ice cold $1 \times$ Hank's balanced salt solution (HBSS) without calcium and magnesium (Gibco, Grand Island, NY, USA; catalog number 14185). Whole brains were removed and sliced into several pieces prior to homogenization with a Dounce homogenizer and/or trituration using a $5 \mathrm{ml} \mathrm{pi-}$ pet. Samples were further triturated using a 20-gauge needle. For some experiments, brain homogenates were further dissociated by incubation in $0.05 \%$ Collagenase D (Roche, Basel, CH; catalog number 11088882 001), 0.09 U/ml Dispase I (Sigma, St. Louis, MO, USA; catalog number D4818) and $0.025 \mathrm{U} / \mathrm{ml}$ DNase I (Sigma, catalog number D4527) in $1 \times$ HBSS at room temperature for 30 minutes with continuous rocking. To isolate the myeloid and immune cells, cells were resuspended in 70\% Percoll/HBSS and fractionated using a Percoll gradient as previously described [27]. Cells were harvested from the 30/70\% interface of the Percoll gradient, washed in HBSS and analyzed by flow cytometry or used for quantitative real-time PCR.

\section{Flow cytometric analysis of CNS populations ex vivo} Following isolation by Percoll gradient, samples were plated onto 96-well plates and analyzed for cell surface markers as previously described [28]. In short, cells were fixed in $2 \%$ paraformaldehyde, then permeabilized in $0.1 \%$ saponin $/ 2 \%$ BSA $/ 1 \times$ PBS. Samples were incubated in an $\mathrm{F}_{\mathrm{C}}$ blocking solution containing rat anti-mouse CD16/CD32 Fcy III/II antibody (BD Pharmingen, San Diego, CA, USA; catalog number 553142) in $2 \%$ donkey serum $/ 0.1 \%$ saponin $/ 2 \% \mathrm{BSA} / 1 \times \mathrm{PBS}$. Cells were incubated with fluorochrome-conjugated antibodies at room temperature. After washing twice, cells were resuspended in PBS and analyzed on a FACSAria flow cytometer (BD Biosciences, San Jose, CA, USA) using FACSDiva software (BD Biosciences, San Jose, CA, USA). Cells were gated as described in the figure legends.

\section{Results}

Neuroinflammatory responses are increased in neonates compared with weanling mice

To determine if the neuroinflammatory response changes in the first weeks of life, we inoculated neonatal (two dayold) and weanling (21 day-old) mice ic with either the TLR4 agonist, LPS, or the TLR9 agonist, CpG-ODNs. Control mice were inoculated with vehicle controls.

Cytokine protein levels were examined at 12 hours post inoculation (hpi), a time point previously shown to be the peak of cytokine production following TLR ligand inoculation in the CNS $[29,30]$. Twelve cytokines were detectable at significant levels following TLR stimulation of the CNS. Several of these cytokines, including IL- $1 \alpha$, IL-1 $\beta$, IL-2, IL-5, IL-6, TNF, CC chemokine ligand (CCL) 2, CCL3 and CXCL9 were significantly higher in neonatal brain tissue than in weanling brain tissue in response to LPS (Figure 1). CpG-ODN also induced higher responses in neonates than in weanling mice for IL-2, IL-5, TNF, CCL2 and CXCL9. No significant agedependent differences were observed for IL-12 or CXCL1. The cytokines tested were undetectable at later time points of 24,48 or 72 hours for both neonates and weanling mice. Overall, the levels of several cytokines induced by TLR4 and/or TLR9 ligand induction were significantly higher in neonates than those observed in weanlings.

Since a difference in the expression of several cytokines was observed between weanling and neonates, we further examined neuroinflammatory responses using real-time PCR analysis. RNA was analyzed at $6 \mathrm{hpi}$, a timepoint that correlates with peak mRNA expression of cytokines following TLR stimulation both in vitro and in vivo $[28,30]$. Analysis of mRNA expression also indicated similarly increased levels of several of these cytokines in neonatal mice as well as increased expression of inducible nitric oxide (Nos2, iNos) mRNA and adhesion molecule Icam1 mRNA (Figure 2, data not shown). In contrast, expression of Csf2 (GM-CSF) mRNA, a growth factor for monocytes, was elevated in brain tissue from 


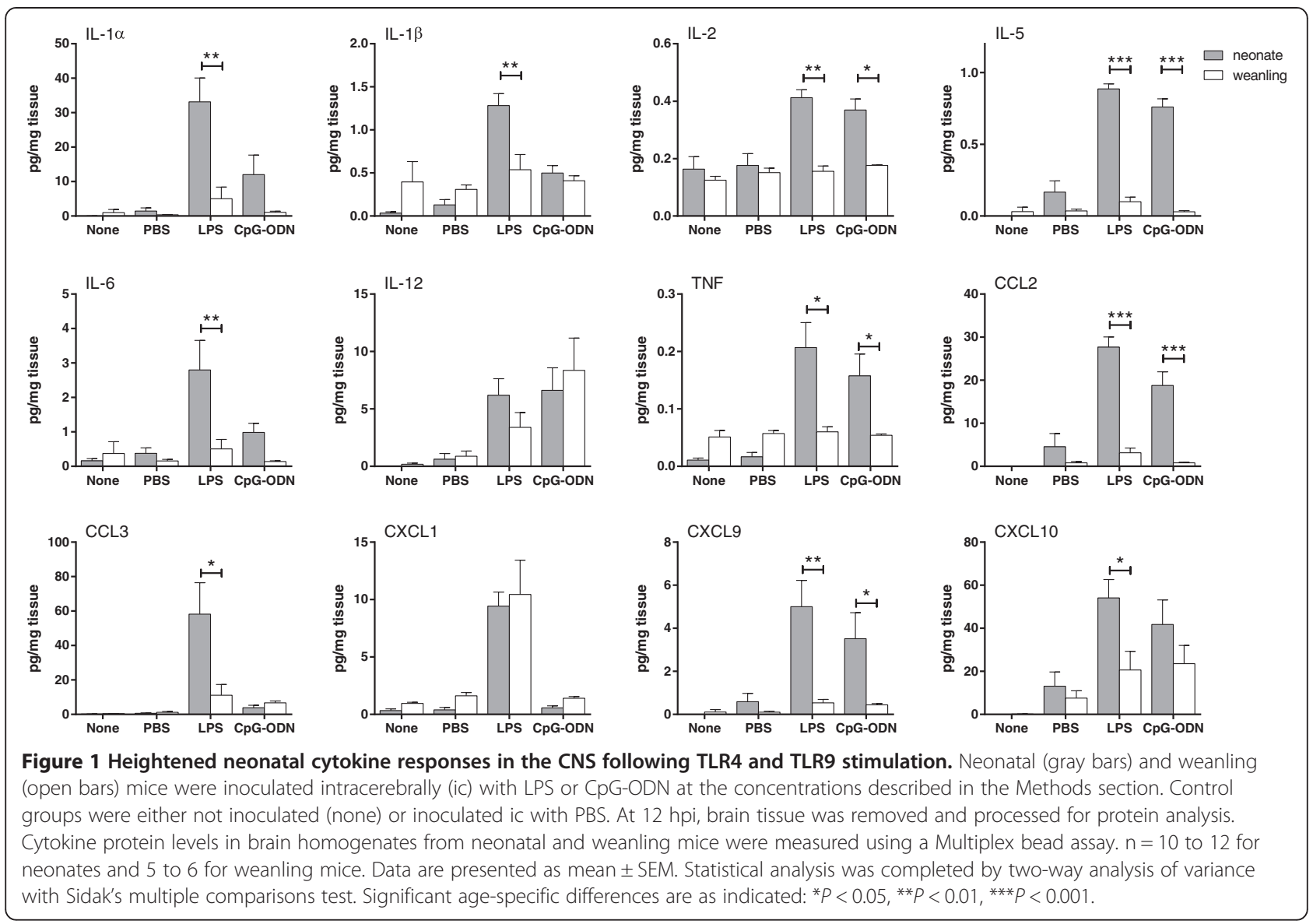

weanling mice compared with neonatal mice (Figure 2). Thus, LPS inoculation induced different neuroinflammatory responses in neonates compared with weanlings with increased production of several cytokines and decreased expression of Csf 2 mRNA.
To examine the time course of these neuroinflammatory responses, we measured mRNA expression levels from 2 to 48 hpi after treatment with either LPS or vehicle control. Modest increases in gene expression for Ccl2 and Ccl3 mRNA were observed in vehicle control-

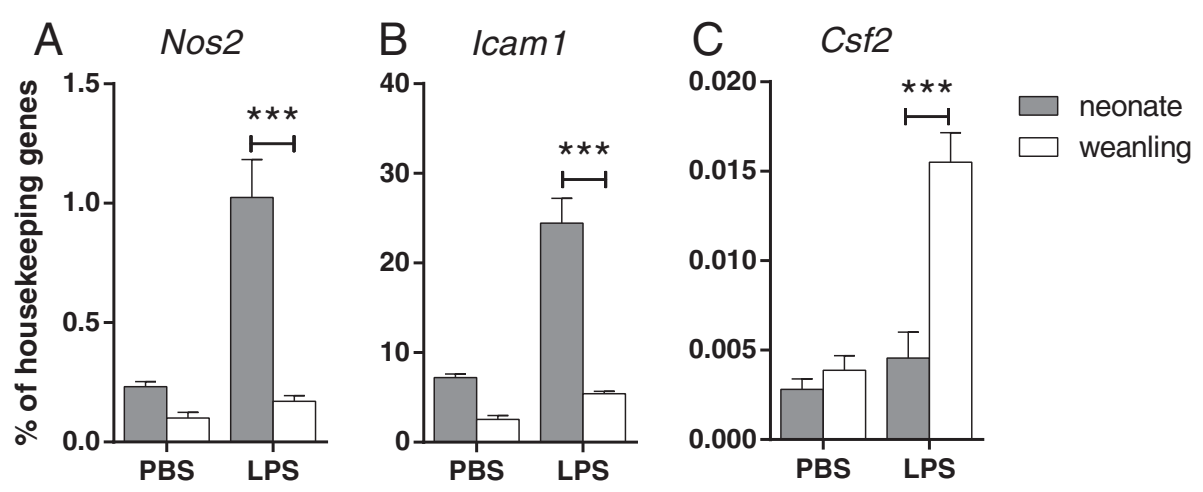

Figure 2 Increased mRNA expression of inflammatory markers in neonatal brain tissue following LPS stimulation. Real-time PCR analysis was used to analyze the expression of neuroinflammatory markers in brain tissue from neonatal and weanling mice at 6 hpi. Increased mRNA expression of Nos2 (iNos) (A) and ICaml (B) mRNAs were observed in brain tissue from LPS-inoculated neonatal mice, while Csf2 (GMCSF) mRNA (C) was elevated in brain tissue from LPS-inoculated weanling mice. $n=4$ mice per group. Data are presented as mean \pm SEM. Statistical analysis was completed by two-way analysis of variance with Tukey's multiple comparisons test. Significant age-specific differences in the LPS response are indicated in the figure as: ${ }^{* *} P<0.001$. 
treated animals for both neonatal and weanling brains at $2 \mathrm{hpi}$ but not at other time points. At $2 \mathrm{hpi}$ after stimulation with LPS, Ccl2 and Il6 mRNA levels were comparable between the two ages, while $C c l 3$ mRNA was higher in neonates (Figure 3). However, by $6 \mathrm{hpi}$, these LPS responses in weanling mice had dropped to nearly basal levels while neonatal $I l 6$ and $C c l 2$ levels remained elevated (Figure 3). Thus, expression of certain cytokine mRNAs were generally higher in neonates and peak expression occurred later than that observed in weanlings. This difference in kinetics may contribute to the increased protein levels of these cytokines observed at 12 hpi (Figure 1).

One possible explanation for the increased neuroinflammatory responses in neonatal mice would be higher expression of TLRs in the brains of neonates than adult mice. However, analysis of mRNA demonstrated lower levels of Tlr4 and Tlr9 mRNA in neonatal brain compared with other ages (Figure 4). Thus, the increased neuroinflammatory response is not likely to be due to increased expression of TLRs in the neonatal brain.

\section{Differences in immune cell markers between neonates and weanlings}

To investigate which cell types might be contributing to the heightened neonatal inflammatory response, we compared mRNA expression levels of inflammatory cells and glial cells in brain tissue from neonatal and weanling mice. Analysis of gene expression for inflammatory cells including $\mathrm{T}$ cells $(C d 3 \varepsilon, C d 8 \alpha)$ and neutrophils (Ela2) showed that these genes were only induced at 12 to 48 hpi (Figure 5A-C), after the peak cytokine response (Figure 3). Thus, an influx of $\mathrm{T}$ cells or neutrophils would probably be responding to, rather than producing, early increases in cytokine levels. The level of mRNA for the astrocyte activation marker Gfap was lower in neonatal brain than weanling brains (Figure 5D), suggesting that more activated astrocytes were also not likely to be the source of heightened cytokine responses in neonates. However, mRNAs for activation markers of myeloid cells (for example, microglia and macrophages), namely Itgax (Cd11c) and Cd80, were elevated at 6 to 12 hpi in neonates compared with weanlings (Figure 5E-F), in concert with the increases in the various cytokine mRNAs. Interestingly, at these time points we also observed elevated mRNA levels for Slamf7 (Figure 5G), whose expression has been reported on natural killer (NK) cells and other immune cells $[31,32]$. Thus, the only cell markers that correlated with the early neuroinflammatory response in neonates were those thought to be primarily for myeloid cells and/or NK cells.

\section{Myeloid cells from brain tissue of neonatal mice have heightened responses to LPS stimulation}

Since resident myeloid cells and/or inflammatory cells such as NK cells could be contributing to the cytokine response, we isolated these cells from the brains of neonatal and weanling mice using a Percoll gradient. The 6 hpi time point was used to allow for RNA analysis of isolated cell fractions. We analyzed cells from the 30/ $70 \%$ interface, which are predominantly microglia, macrophages and infiltrating immune cells [33]. Analysis of this cell fraction for CD4+ $\mathrm{T}$ cells, CD8+ T cells, NK cells and $\mathrm{B}$ cells indicated no significant populations of these cells in the brain for either neonatal or weanling mice (data not shown). Instead, the primary cells in this fraction for both neonates and weanling mice were CD45+ CD11b + myeloid cells; primarily microglia, monocytes and resident macrophages (data not shown).

RNA analysis of the myeloid cell fraction for genes analyzed in whole brain fractions demonstrated greater upregulation of Ccl2, Nos 2 and Slamf7 mRNA following LPS stimulation in neonates compared with weanlings (Figure 6A-C). However, other cytokines, such as Il6,
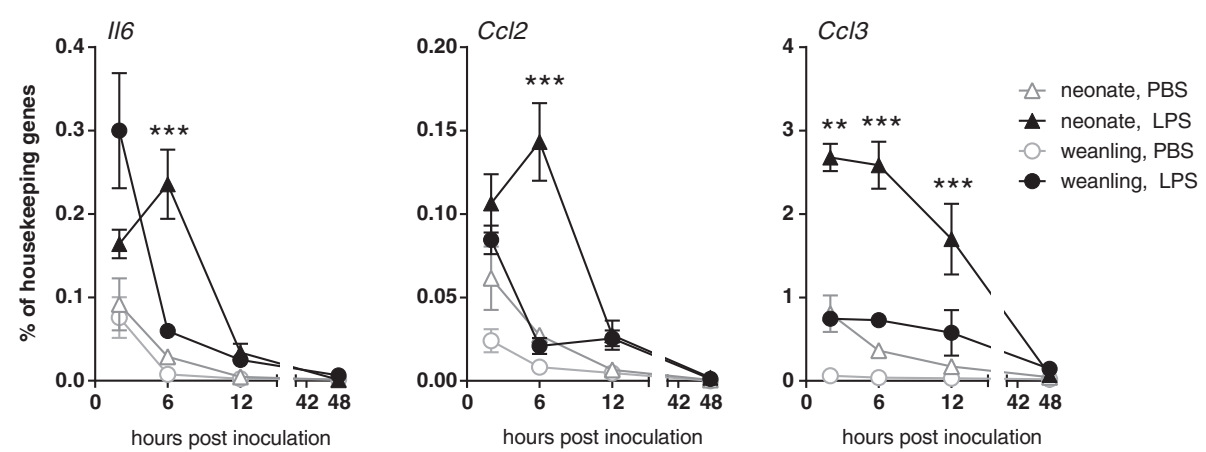

Figure 3 Time course analyses of cytokine mRNA expression indicate differences in the brain cytokine response between neonates and weanlings. Cytokine mRNA levels in brains from neonatal and weanling mice were assayed at 2, 6, 12 and 48 hpi following ic inoculation with PBS or LPS. $\mathrm{n}=4$ to 6 mice per group per time point from combined experiments. Data are presented as mean \pm SEM. Statistical analysis was completed by two-way analysis of variance with Tukey's multiple comparisons test. Significant age-specific differences in the LPS response between neonates and weanlings are indicated: ${ }^{* *} P<0.01,{ }^{* *} P<0.001$. 

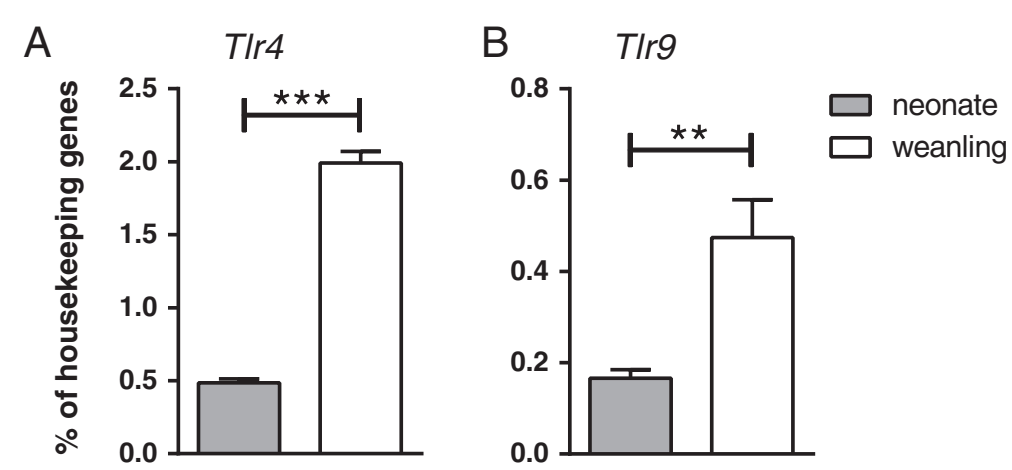

Figure 4 TIr4 and TIr9 mRNA expression is influenced by age. T/r4 (A) and T/r9 (B) mRNA levels were measured in the brains from untreated neonatal and weanling mice. Housekeeping gene expression was averaged using Gusb, Actb and Rp/32. Data are presented as mean \pm SEM ( $n=5$ to 6 per group). Statistical analysis was completed by an unpaired $t$-test. ${ }^{* *} P<0.01,{ }^{* * *} P<0.001$.

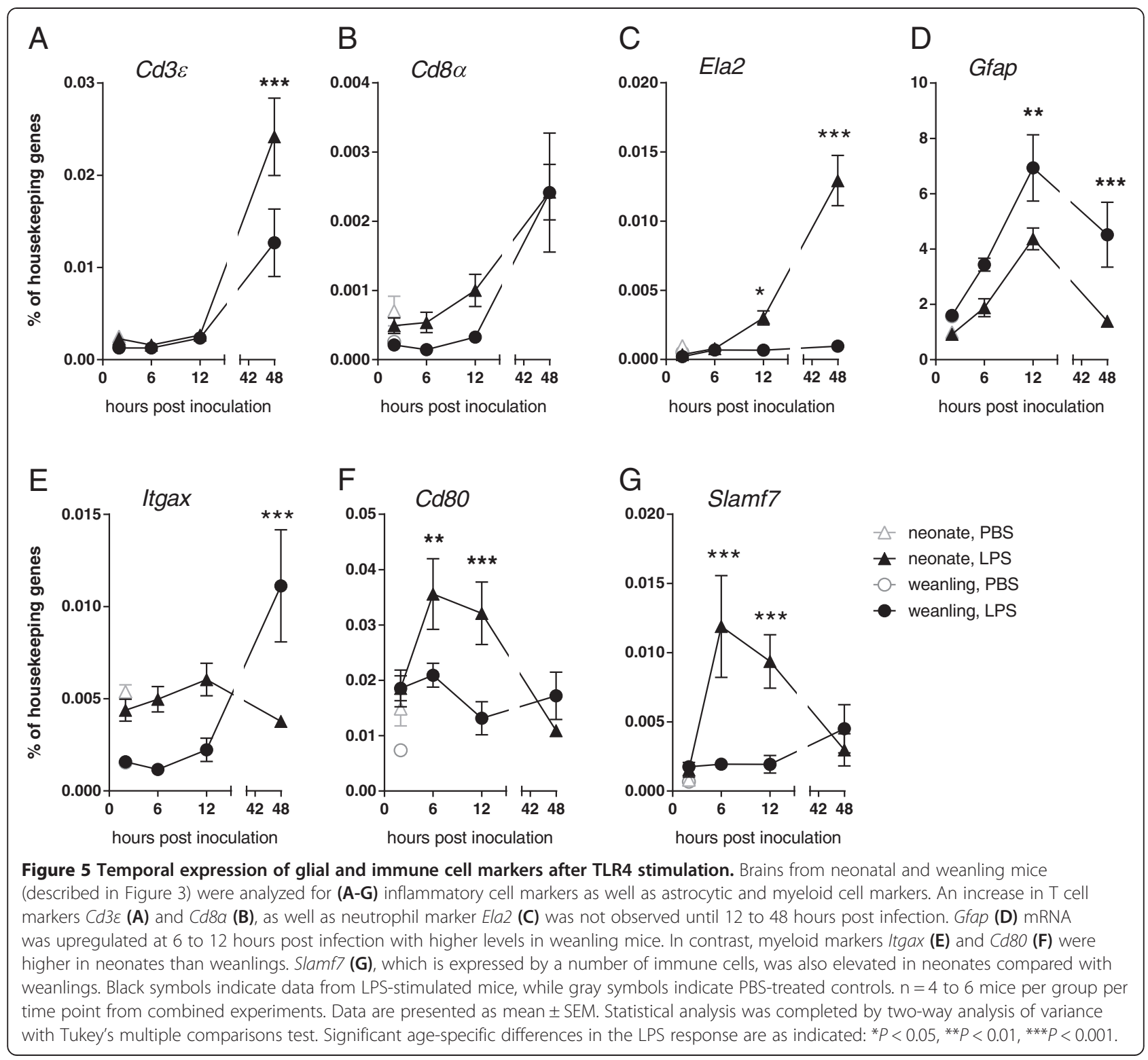



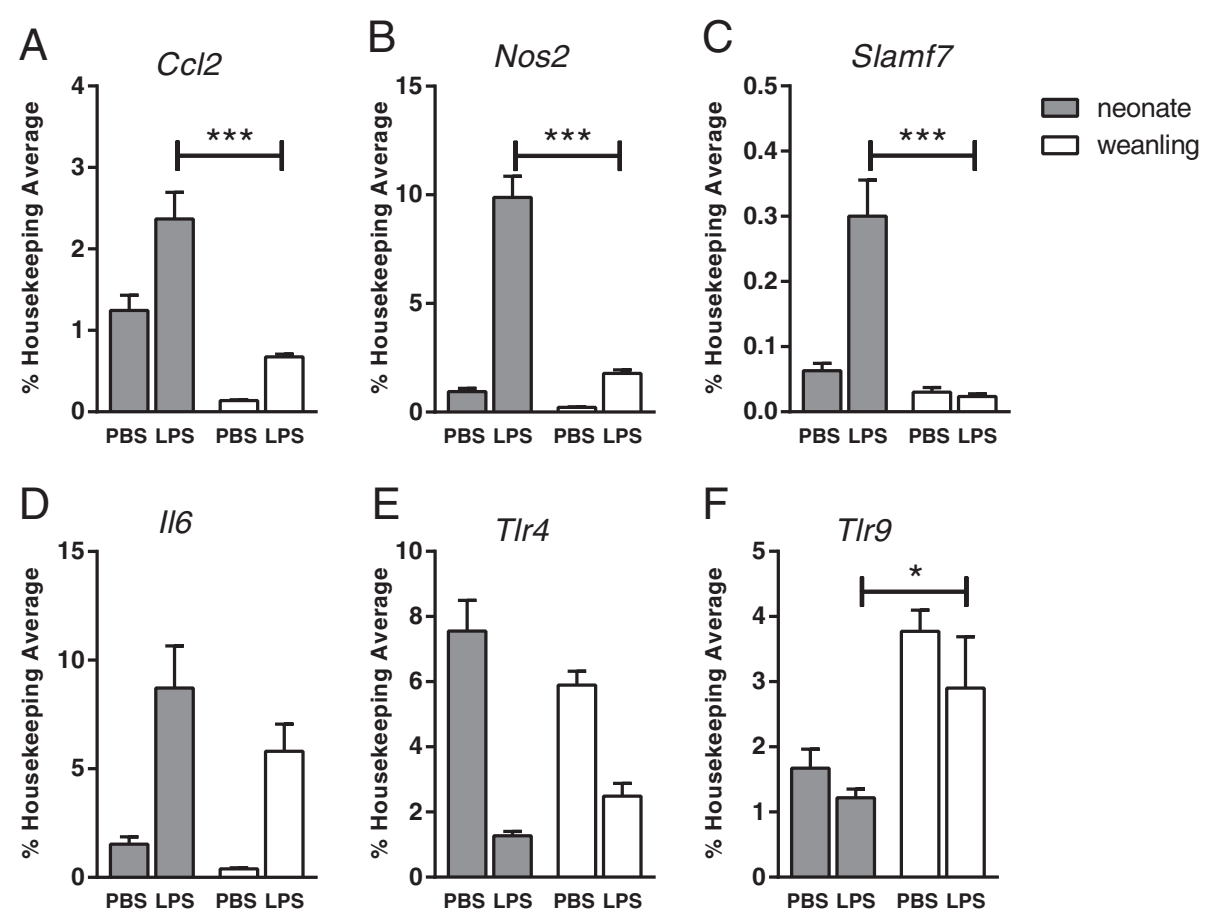

Figure 6 Neonatal versus weanling expression of neuroinflammatory genes in myeloid cells isolated from the CNS. At six hours after ic inoculation with PBS or LPS, brain homogenates from neonatal and weanling mice were fractionated on Percoll gradients, and mRNA expression levels were analyzed by qRT-PCR. mRNA expression of neuroinflammatory markers C 12 (A), Nos2 (B) and Slamf7 (C) were elevated in the myeloid fraction from LPS-inoculated neonatal mice. $/ / 6$ (D) and T/r4 (E) mRNA were not significantly different between age groups, while T/r9 (F) mRNA levels were increased in myeloid cells from weanling mice. $n=4$ to 5 mice per group from combined experiments. Data are presented as mean \pm SEM. Statistical analysis was completed by two-way analysis of variance with Tukey's multiple comparisons test. Significant age-specific-differences in the response to LPS are shown: ${ }^{* * *} P<0.001$.

that were upregulated in neonates at $6 \mathrm{hpi}$ in whole brain tissue (Figure 3) were not significantly different in the myeloid cell fractions at this time point (Figure 6D). Thus, myeloid cells isolated from the brain tissue of neonatal mice had increased upregulation of some of the same proinflammatory genes that were upregulated in the whole brain, indicating that these cells were likely contributing to the increased neuroinflammatory response in neonatal mice.

Interestingly, analyses of Tlr4 and Tlr9 mRNA expression in the myeloid cell fractions indicated no significant difference in Tlr4 mRNA expression in the myeloid fraction from neonatal and weanling mice, while a slight increase in $\operatorname{Tl} 9$ mRNA was observed in weanlings (Figure 6E-F). Thus, the difference in the cytokine response between neonatal and weanling myeloid cells did not correlate with differences in TLR mRNA expression.

\section{Differences in the myeloid cell populations in the brains of neonatal and weanling mice}

Since RNA analysis of myeloid cells from neonatal and weanling mice indicated differences in their neuroinflammatory responses, we further analyzed these cells by flow cytometry at $6 \mathrm{hpi}$, the time point that mRNA differences were observed. Analysis of cells expressing leukocyte common antigen CD45 and the myeloidspecific marker CD11b identified four distinct cell populations. These included $\mathrm{CD} 45^{\mathrm{lo}}, \mathrm{CD} 11 \mathrm{~b}^{\mathrm{lo}}$ microglia (mic, dark blue gate), and three populations of CD $45^{\text {hi }}$ cells that varied in their expression of CD11b (light blue, green and fuchsia gates) (Figure 7). Further analysis using the inflammatory monocyte marker Ly6C indicated that these three cell populations were either Ly6C + monocytes $(\mathrm{m} 1$ green and $\mathrm{m} 2$ fuchsia gates) or $\mathrm{Ly}^{-} \mathrm{C}^{-}$resident macrophages (mac, light blue gate). The monocyte populations have two distinct profiles in the weanling mice which differed in the expression of $\mathrm{CD} 11 \mathrm{~b}$ and $\mathrm{F} 4 / 80$, being either $\mathrm{CD} 11 \mathrm{~b}^{\text {int }}, \mathrm{F} 4 / 80^{\text {hi }}$ ( $\mathrm{m} 1$, green gate) or CD11b ${ }^{\text {hi }}$ and F4/80 (m2, fuschia gate). Lower numbers of all cell populations were observed in neonates compared with weanlings; most notably, the microglia population (mic, dark blue gate) had five-fold fewer cells in neonates than weanlings (Figure 7). In addition, microglia had higher levels of F4/80 (middle panel, $\mathrm{X}$ axis) compared with weanlings indicating that these cells change phenotypically between these two ages. Of the $\mathrm{CD} 45^{\mathrm{hi}}$ populations, the resident macrophage (mac, light blue gate) was also reduced in neonates and was barely detectable. 

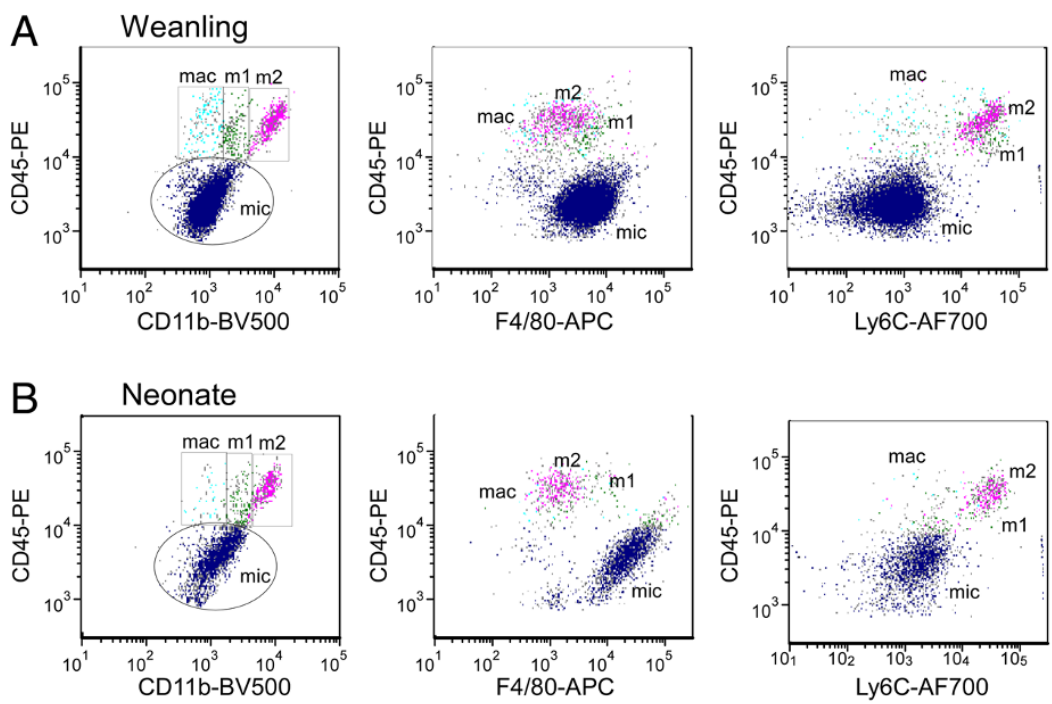

Legend:

$\begin{array}{lllllll}\text { Cell population } & \text { Gate } & \underline{\text { Gate color }} & \underline{\mathrm{CD} 45} & \underline{\mathrm{CD} 11 \mathrm{~b}} & \underline{\mathrm{F} 4 / 80} & \underline{\text { Ly6 }} \\ \text { microglia } & \text { mic } & \text { dark blue } & \text { low } & \text { low } & \text { high } & \text { low } \\ \text { macrophage } & \text { mac } & \text { light blue } & \text { high } & \text { low } & \text { low } & \text { lo } \\ \text { monocyte 1 } & \mathrm{m} 1 & \text { green } & \text { high } & \text { int. } & \text { high } & \text { high } \\ \text { monocyte 2 } & \mathrm{m} 2 & \text { fuschia } & \text { high } & \text { high } & \text { low } & \text { hig }\end{array}$

$$
\begin{array}{cc}
\begin{array}{c}
\text { number of cells (average }+/ \text { - SEM) } \\
\text { weanling } \\
\text { neonate }
\end{array} \\
11,341+/-1,860 & 2,112+/-205 \\
179+/-28 & 52+/-13 \\
282+/-107 & 131+/-18 \\
608+/-111 & 456+/-101
\end{array}
$$

Figure 7 Differences in the microglial and resident macrophage populations in the CNS of neonatal and weanling mice. Cells from weanling (A) and neonatal (B) mice at 6 hpi with LPS were isolated on Percoll gradients and the myeloid cell fraction was collected from the 30/70\% interface and analyzed by flow cytometry. An initial gate was drawn based on forward and side scatter to exclude cellular debris and doublets. This cell fraction was further gated on only CD45+ and CD11b + cells to directly analyze the myeloid cells. The CD45+ CD11b + cells were subsequently divided into subpopulations based on their relative expression of CD11b and CD 45. These included a CD45 $5^{\circ}, C D 11 \mathrm{~b}+$ microglial population (dark blue gate) and three populations of CD $45^{\text {hi }}$ cells: CD1 $1 b^{\text {lo }}$ (light blue), CD11 b ${ }^{\text {int }}$ (green) and CD1 1 b ${ }^{\text {hi }}$ (fuchsia). These populations were then analyzed using CD45 and F4/80 or CD45 and Ly6C to identify resident macrophage and monocyte populations. Data are representative of 4 to 5 mice per group.

To examine the functional state of these cell types, we examined cell surface markers related to cell activation: CD11a (integrin $\alpha \mathrm{L}$ ) and CD86; as well as those associated with inhibition of activation: CD200R (OX2R) and $\mathrm{CD172a}$ (SIRP $\alpha)$ [34-37]. Cells were analyzed at $6 \mathrm{hpi}$, that is, at the peak of cytokine mRNA expression. A significant difference was observed between neonatal and weanling mice in the mean fluorescent intensity (MFI) for these molecules (Figure 8), although the relative surface expression of these molecules was not altered by LPS stimulation (data not shown). Neonatal microglia had higher levels of CD11a and CD172a, but lower levels of CD86 (Figure 8). Neonatal monocytes had lower levels of CD11a than weanling monocytes, with varying levels of CD86, CD200R and CD172a depending on the subpopulation (Figure 8 ). Thus, there were distinct differences in the expression of cell surface regulatory markers between neonates and weanlings, but these varied substantially between the cell populations.
SLAMF7 is expressed at high levels in neonatal microglia Slamf7 was one of the markers whose mRNA expression was upregulated in whole brain tissue and in the myeloid/immune cells fraction from neonatal mice (Figure 5G, Figure 6C). SLAMF7 is often used as an activation marker for NK cells $[31,32,38]$, however, we were unable to detect NK cells in the CNS by flow cytometry (data not shown). Although SLAMF7 was recently detected on activated human monocytes [39], expression in the CNS has not been reported. Analysis of SLAMF7 on the myeloid cells in the CNS demonstrated clear expression of SLAMF7 on neonatal microglia and lower levels of SLAMF7 on weanling microglia (Figure 9A), but was not upregulated following LPS stimulation (Figure 9B). SLAMF7 was not expressed at levels above background on the monocyte or macrophage population (Figure 9C, data not shown) for either PBS or LPS inoculated mice. Thus, SLAMF7 appears to be primarily expressed by neonatal microglia and may be a useful 

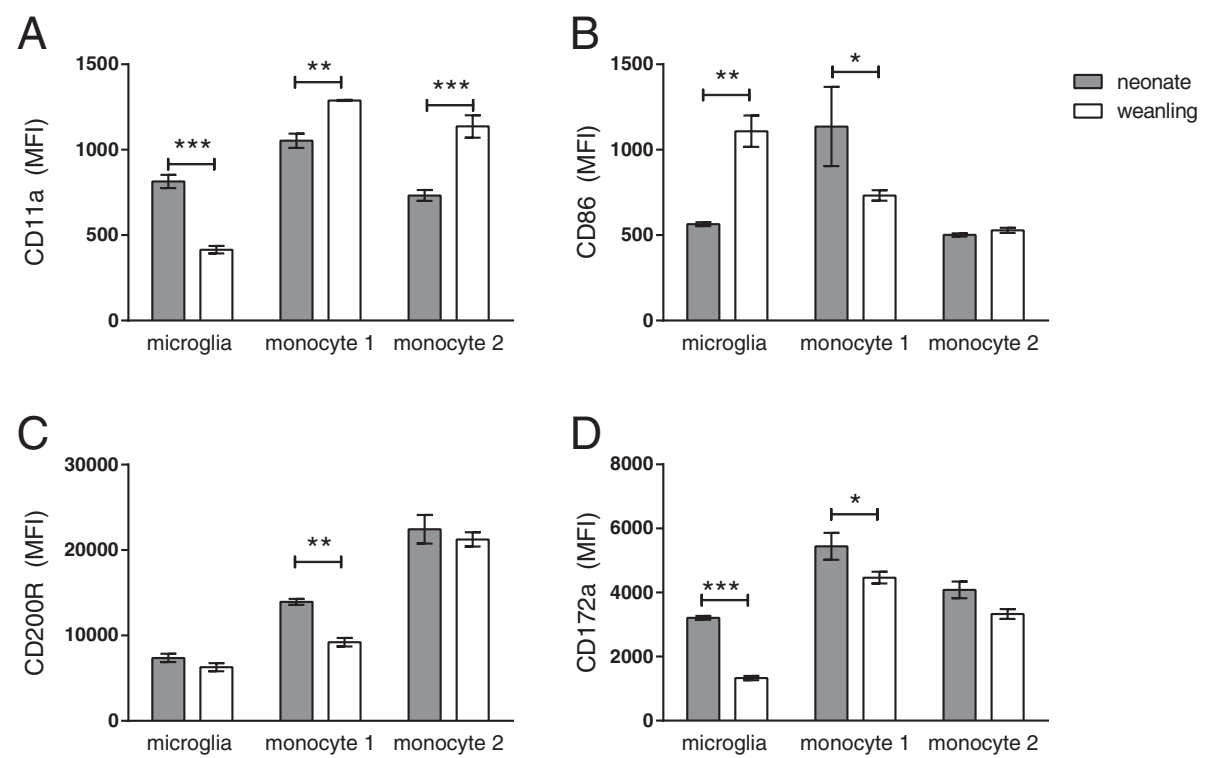

Figure 8 Neonatal versus weanling expression of activating and inhibitory markers on microglia. Cells shown in Figure 7 were additionally analyzed for expression of activation markers CD11a (A) and CD86 (B) as well as inhibitory markers CD200R (C) and CD172a (D). Mean Fluorescent Intensity (MFI) of microglia $\left(C D 45^{10}, C D 11 b^{\text {lo }}\right.$ dark blue gate in Figure 7$)$ and both monocyte populations are shown. Monocyte 1 population contained cells that were CD11 ${ }^{\text {int }}, F 4 / 80^{\text {hi }}$, Ly6C + (green gate in Figure 7) while monocyte 2 population contained cells that were $\mathrm{CD} 11 \mathrm{~b}{ }^{\mathrm{hi}}, \mathrm{F} 4 / 80^{\circ}$, Ly6C + (fuchsia gate in Figure 7). Resident macrophages (light blue gate in Figure 7) were not analyzed since there were not sufficient cells in the neonatal cell population for comparison. No difference was observed between the PBS and LPS treated groups for any marker (data not shown). Data are the mean \pm SEM for 3 mice per group. Data were similar from two experiments, although different antibody concentrations were used in the second experiment (not shown). Statistical analysis was completed by two-way analysis of variance with Tukey's multiple comparisons test. Significant age-specific differences are as indicated: ${ }^{*} P<0.05$, ${ }^{*} P<0.01,{ }^{* *} P<0.001$.

marker for identifying this population. The higher expression of SLAMF7, CD11a, F4/80 and CD172a on neonatal microglia compared with weanling microglia indicates a clear phenotype on these cells that correlates with the heightened inflammatory response in the CNS.

\section{Discussion}

In the current study, we observed heightened responses to TLR stimulation in the CNS in neonatal mice compared with weanlings. This response was most notable with LPS, but was also observed with CpG-ODNs. Kinetic analysis of gene expression indicated that the increased cytokine response in neonates peaked at $6 \mathrm{hpi}$, which was associated with an increase in activation markers for myeloid cells in the CNS. Analysis of this cell population in the brains of neonatal and weanling mice indicated two primary differences in the myeloid population between these ages. The first was more macrophages in weanling mice and the second was increased expression of multiple regulatory molecules on neonatal microglia. Surprisingly, SLAMF7, a regulatory molecule on NK cells [32,38], was detected on neonatal, microglia and the mRNA expression of this molecule tightly correlated with cytokine induction.

The increased cytokine expression following TLR stimulation in neonates in the brain is opposite of the activation of monocytes/macrophages in the periphery. For example, neonatal mouse monocytes/macrophages have reduced cytokine responses to TLR4 stimulation compared with adults, with the exception of IL-10 production [40,41]. Similar age-related effects were also observed in studies with human monocytes/macrophages $[12,16,18,19]$. The primary reason for the differences in the periphery compared with the CNS may simply be the requirement for microglia in the CNS to be active in neonates for synaptic pruning and other developmental processes. A similar role in development has not been reported for peripheral monocytes. Thus, unlike adult animals in which the immune response in the CNS is considered to be more limited than in peripheral tissues, the CNS in neonatal mice may be more responsive to insult or injury and produce a more substantial inflammatory response. Since development of the neonatal mouse brain corresponds in several parameters with the second trimester development of the human brain $[10,42]$, this time period in humans may be particularly sensitive to immune stimulation. However, since myeloid cells appear to be responsible for this increased inflammatory response, the inflammatory response in the human brain will most likely correlate with the timeframe in which these cells are most active during development, which may differ from that observed in mice. 

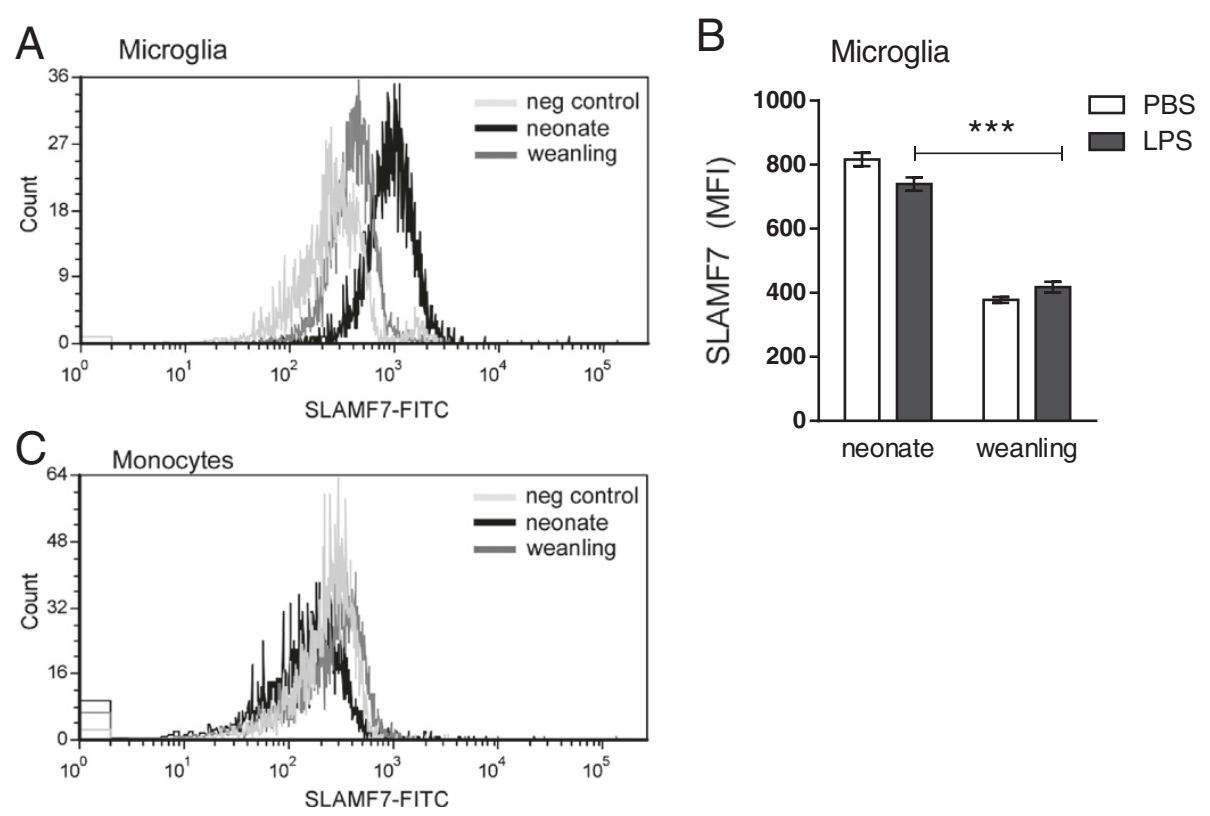

Figure 9 SLAMF7 is strongly expressed by neonatal microglia. Cells were isolated as described in Figure 7. (A) SLAMF7 expression on microglia cells (dark blue gate in Figure 7) from an LPS-inoculated neonatal mouse (black line) or weanling mouse (gray line). Light gray line is from a sample that did not contain the anti-SLAMF7 antibody (no antibody negative control). Plots shown are from a single mouse but are consistent with data from 2 to 4 mice per age. (B) Average MFI of SLAMF7 expression on microglia cells from PBS or LPS-treated mice showing a higher level of SLAMF7 on neonatal microglia. Data are the mean \pm SEM for 3 LPS-treated mice per group at 6 hpi. Only two data points are plotted for PBS-treated controls and thus represent a range of data rather than SEM. Data are representative of two replicate experiments, although different antibody concentrations were used in the second experiment (not shown). ${ }^{* *} P<0.001$ for direct comparison of LPS-treated groups (C) SLAMF7 expression was not detected on the monocyte populations (combined cells from green and fuchsia gates in Figure 7) when compared with the negative (no antibody) control (light gray line).

The elevated response by the myeloid population in the neonatal CNS is surprising considering the lower level of $\mathrm{Tlr}$ mRNA expression and the higher levels of inhibitory molecules, including CD200R and CD172a (Figure 4, Figure 8). Deficiency in CD200R has been linked to increased inflammation in models of experimental autoimmune encephalitis (EAE) and Parkinson's disease $[43,44]$, while CD172a negatively regulates CD11b-mediated adhesion, migration and phagocytosis $[35,45]$. The higher expression of these receptors would predict a reduced responsiveness to TLR stimulation. However, the functional ability of CD172a and CD200R to inhibit myeloid cell activation may be dependent on many factors, including expression of their ligands, CD47 and CD200 respectively, on surrounding cells, as well as the expression of signaling molecules necessary to mediate inhibition of activation. Thus, the expression of these receptors may not be sufficient to diminish the inflammatory response to TLR stimulation in the neonatal brain.

The presence of SLAMF7 on neonatal microglia may be an additional negative regulator of microglia activation. SLAMF7 is a self-ligand and is involved in both cell activation as well as cell inhibition for NK cells [32]. However, analysis of activated human monocytes indicated that these cells did not express EAT-2, which is required for SLAMF7-induced activation [39]. SLAM F7 inhibited MAPK activity of monocytes [39], suggesting that SLAMF7 may function as a negative regulator of myeloid cell activation. It is possible that SLAMF7 may function in a manner similar to CD200R or CD172a and play a critical role in controlling the function of microglia and their interactions with neurons in the developing brain.

In addition to the elevated expression of negative regulators, neonatal microglia also had higher levels of integrins, including CD11a and CD11b. CD11b may be involved in TLR4 signaling by influencing the recruitment and degradation of intracellular TLR signaling adaptor proteins [46,47]. Therefore, elevated microglial CD11b levels could alter TLR4 signaling in neonatal microglia, resulting in the elevated cytokine responses observed in the CNS of neonatal mice.

The ratio of the $\mathrm{Ly} 6 \mathrm{C}^{\mathrm{hi}} \mathrm{CD} 11 \mathrm{~b}^{\mathrm{hi}} \mathrm{F} 4 / 80^{\text {lo }}$ monocytes ( $\mathrm{m} 2$, fuschia gate) relative to the other three myeloid cell populations was higher in neonates than in weanling mice, although the relative number of cells $\mathrm{m} 2$ was comparable between age groups (Figure 7). The increased ratio of this cell population may indicate that this cell population is a contributor to the inflammatory response 
in neonates. Additionally, the reduced ratio of the other three cell populations could influence the inflammatory response if these cells had a regulatory role in limiting inflammation through the production of anti-inflammatory cytokines or inhibitory molecules. We did not observe any increase in IL-10 production in the weanling brain compared with the neonatal brain, indicating that these macrophages were not alternatively activated macrophages (data not shown). However, we cannot rule out localized production of inhibitory cytokines by these cells that would limit microglial or monocyte activation and cytokine production.

One of the few cytokines that was elevated in the weanling brain compared with the neonatal brain was Csf2 (GMCSF) a cytokine known to be involved in the activation and maintenance of microglia and macrophages [48]. This induction of Csf 2 mRNA could be due to the higher percentages of the monocyte/macrophage populations in the weanling mice. Additionally, quiescent microglia may be programmed to produce more GMCSF upon stimulation to support cell activation and proliferation, while the neonatal amoeboid microglia are already in a heightened activation state.

Our studies indicate that myeloid cells are at least partially responsible for the increased proinflammatory response observed in neonates. However, other cell types including endothelia, oligodendroglia, neurons and astrocytes may also contribute to the differences in the cytokine production. Astrocytes have also been shown to produce cytokines in response to TLR stimulation, including TLR4 $[28,49,50]$. Astrocytes also undergo developmental changes in the first few weeks of birth, including morphological changes and gene expression [51,52], which may influence their response to TLR stimulation. Interestingly, Gfap mRNA expression, a marker of astrocyte activation, was lower in neonates compared with weanlings (Figure 5D). Thus, astrocytes may contribute to the neuroinflammatory response in both weanlings and neonates, but are unlikely to be a major source of the increased cytokine response in neonates.

\section{Conclusions}

Although elevated levels of IL-6, IL-1 $\beta$ and TNF in the neonatal brain have been correlated with later cognitive disability and behavioral changes [7], few published studies directly compare the neuroinflammatory response of neonates with that of older animals. In our studies, we demonstrate that neonates have an altered neuroinflammatory response to TLR stimulation compared with older animals. The stronger response of myeloid cells to TLR stimulation following insult or injury to the CNS may have severe consequences in the neonate. Microglial production of soluble factors influence neuronal cell maturation and apoptosis and can affect neuroprogenitor proliferation and differentiation [20,22,53,54]. Stimulation via TLR4 alters the activation state of these cells as observed by the strong cytokine responses and Nos 2 mRNA expression (Figure 1, Figure 2). Thus, increased microglial activation may significantly alter brain development resulting in the long-term damage that has been associated with neonatal insults to the brain. Understanding the factors that regulate this distinct neuroinflammatory response will be important in preventing long-term damage following infection in the neonatal brain.

\section{Abbreviations}

Actb: beta actin; BSA: bovine serum albumin; CCL: CC chemokine ligand; CNS: central nervous system; CpG-ODN: unmethylated CpG oligodeoxynucleotides; CSF2: colony stimulating factor 2 (granulocytemacrophage); CXCL: CXC chemokine ligand; ELA2: neutrophil expressed elastase; GFAP: glial fibrillary acidic protein; GUSB: beta glucuronidase; HBSS: Hank's balanced salt solution; IC: intracerebrally; IL: interleukin; ITGAX: integrin alpha X; LPS: lipopolysaccharide; MFI: mean fluorescent intensity; NK: natural killer; NOS2: inducible nitric oxide; PAMPs: Pathogen Associated Molecular Patterns; PBS: phosphate-buffered saline; PCR: polymerase chain reaction; RPL32: ribosomal protein L32;

SLAMF7: signaling lymphocytic activation molecule family member 7; TLR: Toll-like Receptor; TNF: tumor necrosis factor.

\section{Competing interests}

The authors declare that they have no competing interests.

\section{Authors' contributions}

LC and TW conducted the experiments including injections, removal of tissue, analysis of tissue by RT-PCR and processing of cells for flow cytometry. AC analyzed cells by flow cytometry. LC, BC and KP participated in conceiving and designing of the study. LC, BC and KP drafted the manuscript. All authors read and approved the final manuscript.

\section{Acknowledgements}

The authors would like to thank Bruce Chesebro, Sue Priola, Katherine Taylor and Clayton Winkler for review of the manuscript and helpful suggestions. The authors would also like to thank Lara Myers for her helpful suggestions on flow cytometry. This work was funded by the Intramural Research program of the National Institute of Allergy and Infectious Disease.

Received: 6 February 2014 Accepted: 21 March 2014

Published: 4 April 2014

\section{References}

1. Brown AS, Schaefer CA, Quesenberry CP Jr, Liu L, Babulas VP, Susser ES Maternal exposure to toxoplasmosis and risk of schizophrenia in adult offspring. Am J Psychiatry 2005, 162:767-773.

2. Nelson $K B$, Willoughby RE: Infection, inflammation and the risk of cerebral palsy. Curr Opin Neurol 2000, 13:133-139.

3. Sorensen HJ, Mortensen EL, Reinisch JM, Mednick SA: Association between prenatal exposure to bacterial infection and risk of schizophrenia. Schizophr Bull 2009, 35:631-637.

4. Chen GH, Wang H, Yang QG, Tao F, Wang C, Xu DX: Acceleration of age-related learning and memory decline in middle-aged CD-1 mice due to maternal exposure to lipopolysaccharide during late pregnancy. Behav Brain Res 2011, 218:267-279.

5. Ling Z, Zhu Y, Tong C, Snyder JA, Lipton JW, Carvey PM: Progressive dopamine neuron loss following supra-nigral lipopolysaccharide (LPS) infusion into rats exposed to LPS prenatally. Exp Neurol 2006, 199:499-512.

6. Wang S, Yan JY, Lo YK, Carvey PM, Ling Z: Dopaminergic and serotoninergic deficiencies in young adult rats prenatally exposed to the bacterial lipopolysaccharide. Brain Res 2009, 1265:196-204. 
7. Bilbo SD, Schwarz JM: Early-life programming of later-life brain and behavior: a critical role for the immune system. Front Behav Neurosci 2009, 3:14.

8. Gilmore JH, Jarskog LF: Exposure to infection and brain development: cytokines in the pathogenesis of schizophrenia. Schizophr Res 1997, 24:365-367.

9. Clancy B, Darlington RB, Finlay BL: Translating developmental time across mammalian species. Neuroscience 2001, 105:7-17.

10. Clancy B, Finlay BL, Darlington RB, Anand KJ: Extrapolating brain development from experimental species to humans. Neurotoxicology 2007, 28:931-937.

11. Meyer U, Feldon J, Fatemi SH: In-vivo rodent models for the experimental investigation of prenatal immune activation effects in neurodevelopmental brain disorders. Neurosci Biobehav Rev 2009, 33:1061-1079.

12. Belderbos ME, Van Bleek GM, Levy O, Blanken MO, Houben ML, Schuijff L, Kimpen $J$, Bont L: Skewed pattern of Toll-like receptor 4-mediated cytokine production in human neonatal blood: low LPS-induced IL-12p70 and high IL-10 persist throughout the first month of life. Clin Immunol 2009, 133:228-237.

13. Pedraza-Sanchez S, Hise AG, Ramachandra L, Arechavaleta-Velasco F, King $\mathrm{CL}$ : Reduced frequency of a CD14+ CD16+ monocyte subset with high Toll-like receptor 4 expression in cord blood compared to adult blood contributes to lipopolysaccharide hyporesponsiveness in newborns. Clin Vaccine Immunol 2013, 20:962-971.

14. Liao SL, Yeh KW, Lai SH, Lee WI, Huang JL: Maturation of Toll-like receptor 1-4 responsiveness during early life. Early Hum Dev 2013, 89:473-478.

15. Charrier E, Cordeiro P, Cordeau M, Dardari R, Michaud A, Harnois M, Merindol N, Herblot S, Duval M: Post-transcriptional down-regulation of Toll-like receptor signaling pathway in umbilical cord blood plasmacytoid dendritic cells. Cell Immunol 2012, 276:114-121.

16. Burl S, Townend J, Njie-Jobe J, Cox M, Adetifa UJ, Touray E, Philbin VJ, Mancuso C, Kampmann B, Whittle H, Jaye A, Flanagan KL, Levy O: Age-dependent maturation of Toll-like receptor-mediated cytokine responses in Gambian infants. PLoS One 2011, 6:e18185.

17. PrabhuDas M, Adkins B, Gans H, King C, Levy O, Ramilo O, Siegrist CA Challenges in infant immunity: implications for responses to infection and vaccines. Nat Immunol 2011, 12:189-194.

18. Levy O, Zarember KA, Roy RM, Cywes C, Godowski PJ, Wessels MR: Selective impairment of TLR-mediated innate immunity in human newborns: neonatal blood plasma reduces monocyte TNF-alpha induction by bacterial lipopeptides, lipopolysaccharide, and imiquimod, but preserves the response to R-848. J Immunol 2004, 173:4627-4634.

19. De WD, Tonon S, Olislagers V, Goriely S, Boutriaux M, Goldman M, Willems F: Impaired responses to toll-like receptor 4 and toll-like receptor 3 ligands in human cord blood. J Autoimmun 2003, 21:277-281.

20. Ghosh A, Streit WJ, Minghetti L, Basu A: Microglia in development and disease. Clin Dev Immunol 2013, 2013:736459.

21. Harry GJ: Microglia during development and aging. Pharmacol Ther 2013 139:313-326.

22. Lewis S: Development: microglia go pruning. Nat Rev Neurosci 2011, 12:492-493.

23. Alliot F, Godin I, Pessac B: Microglia derive from progenitors, originating from the yolk sac, and which proliferate in the brain. Brain Res Dev Brain Res 1999, 117:145-152.

24. Lewis SD, Butchi NB, Khaleduzzaman M, Morgan TW, Du M, Pourciau S, Baker DG, Akira S, Peterson KE: Toll-like receptor 7 is not necessary for retroviral neuropathogenesis but does contribute to virus-induced neuroinflammation. J Neurovirol 2008, 14:492-502.

25. Livak KJ, Schmittgen TD: Analysis of relative gene expression data using real-time quantitative PCR and the 2(-Delta Delta $C(T))$ Method. Methods 2001, 25:402-408.

26. Schmittgen TD, Livak KJ: Analyzing real-time PCR data by the comparative C (T) method. Nat Protoc 2008, 3:1101-1108.

27. Peterson KE, Evans LH, Wehrly K, Chesebro B: Increased proinflammatory cytokine and chemokine responses and microglial infection following inoculation with neural stem cells infected with polytropic murine retroviruses. Virology 2006, 354:143-153.

28. Butchi NB, Du M, Peterson KE: Interactions between TLR7 and TLR9 agonists and receptors regulate innate immune responses by astrocytes and microglia. Glia 2010, 58:650-664.
29. Butchi NB, Pourciau S, Du M, Morgan TW, Peterson KE: Analysis of the neuroinflammatory response to TLR7 stimulation in the brain: comparison of multiple TLR7 and/or TLR8 agonists. J Immunol 2008, 180:7604-7612

30. Butchi NB, Woods T, Du M, Morgan TW, Peterson KE: TLR7 and TLR9 trigger distinct neuroinflammatory responses in the CNS. Am J Pathol 2011, 179:783-794.

31. Tassi I, Colonna M: The cytotoxicity receptor CRACC (CS-1) recruits EAT-2 and activates the PI3K and phospholipase Cgamma signaling pathways in human NK cells. J Immunol 2005, 175:7996-8002.

32. Veillette A: NK cell regulation by SLAM family receptors and SAP-related adapters. Immunol Rev 2006, 214:22-34.

33. Campanella M, Sciorati C, Tarozzo G, Beltramo M: Flow cytometric analysis of inflammatory cells in ischemic rat brain. Stroke 2002, 33:586-592.

34. Wang XJ, Ye M, Zhang YH, Chen SD: CD200-CD200R regulation of microglia activation in the pathogenesis of Parkinson's disease. J Neuroimmune Pharmacol 2007, 2:259-264.

35. Gitik M, Liraz-Zaltsman S, Oldenborg PA, Reichert F, Rotshenker S: Myelin down-regulates myelin phagocytosis by microglia and macrophages through interactions between CD47 on myelin and SIRPalpha (signal regulatory protein-alpha) on phagocytes. J Neuroinflammation 2011, 8:24

36. Satoh J, Lee YB, Kim SU: T-cell costimulatory molecules B7-1 (CD80) and B7-2 (CD86) are expressed in human microglia but not in astrocytes in culture. Brain Res 1995, 704:92-96.

37. Moneta ME, Gehrmann J, Topper R, Banati RB, Kreutzberg GW: Cell adhesion molecule expression in the regenerating rat facial nucleus. J Neuroimmunol 1993, 45:203-206.

38. Boles KS, Barten R, Kumaresan PR, Trowsdale J, Mathew PA: Cloning of a new lectin-like receptor expressed on human NK cells. Immunogenetics 1999, 50:1-7.

39. Kim JR, Horton NC, Mathew SO, Mathew PA: CS1 (SLAMF7) inhibits production of proinflammatory cytokines by activated monocytes. Inflamm Res 2013, 62:765-772.

40. Chelvarajan L, Popa D, Liu Y, Getchell TV, Stromberg AJ, Bondada S: Molecular mechanisms underlying anti-inflammatory phenotype of neonatal splenic macrophages. J Leukoc Biol 2007, 82:403-416.

41. Chelvarajan RL, Collins SM, Doubinskaia IE, Goes S, Van WJ, Flanagan D, De Villiers WJ, Bryson JS, Bondada S: Defective macrophage function in neonates and its impact on unresponsiveness of neonates to polysaccharide antigens. J Leukoc Biol 2004, 75:982-994.

42. Clancy B, Kersh B, Hyde J, Darlington RB, Anand KJ, Finlay BL: Web-based method for translating neurodevelopment from laboratory species to humans. Neuroinformatics 2007, 5:79-94

43. Meuth SG, Simon OJ, Grimm A, Melzer N, Herrmann AM, Spitzer P, Landgraf $P$, Wiendl $\mathrm{H}$ : CNS inflammation and neuronal degeneration is aggravated by impaired CD200-CD200R-mediated macrophage silencing. J Neuroimmunol 2008, 194:62-69.

44. Zhang S, Wang XJ, Tian LP, Pan J, Lu GQ, Zhang YJ, Ding JQ, Chen SD: CD200-CD200R dysfunction exacerbates microglial activation and dopaminergic neurodegeneration in a rat model of Parkinson's disease. J Neuroinflammation 2011, 8:154.

45. Liu DQ, Li LM, Guo YL, Bai R, Wang C, Bian Z, Zhang CY, Zen K: Signal regulatory protein alpha negatively regulates beta2 integrin-mediated monocyte adhesion, transendothelial migration and phagocytosis. PLoS One 2008, 3:e3291.

46. Han C, Jin J, Xu S, Liu H, Li N, Cao X: Integrin CD11b negatively regulates TLR-triggered inflammatory responses by activating Syk and promoting degradation of MyD88 and TRIF via Cbl-b. Nat Immunol 2010, 11:734-742.

47. Scott MJ, Billiar TR: Beta2-integrin-induced p38 MAPK activation is a key mediator in the CD14/TLR4/MD2-dependent uptake of lipopolysaccharide by hepatocytes. J Biol Chem 2008, 283:29433-29446.

48. Ganter S, Northoff H, Mannel D, Gebicke-Harter PJ: Growth control of cultured microglia. J Neurosci Res 1992, 33:218-230.

49. Holm TH, Draeby D, Owens T: Microglia are required for astroglial Toll-like receptor 4 response and for optimal TLR2 and TLR3 response. Glia 2012, 60:630-638

50. Krasowska-Zoladek A, Banaszewska M, Kraszpulski M, Konat GW: Kinetics of inflammatory response of astrocytes induced by TLR 3 and TLR4 ligation. J Neurosci Res 2007, 85:205-212.

51. Bushong EA, Martone ME, Ellisman MH: Maturation of astrocyte morphology and the establishment of astrocyte domains during postnatal hippocampal development. Int J Dev Neurosci 2004, 22:73-86. 
52. Freeman MR: Specification and morphogenesis of astrocytes. Science 2010, 330:774-778.

53. Antony JM, Paquin A, Nutt SL, Kaplan DR, Miller FD: Endogenous microglia regulate development of embryonic cortical precursor cells. J Neurosci Res 2011, 89:286-298.

54. Pang Y, Campbell L, Zheng B, Fan L, Cai Z, Rhodes P: Lipopolysaccharideactivated microglia induce death of oligodendrocyte progenitor cells and impede their development. Neuroscience 2010, 166:464-475.

doi:10.1186/1742-2094-11-70

Cite this article as: Christensen et al: Age-related differences in neuroinflammatory responses associated with a distinct profile of regulatory markers on neonatal microglia. Journal of Neuroinflammation 2014 11:70

\section{Submit your next manuscript to BioMed Central and take full advantage of:}

- Convenient online submission

- Thorough peer review

- No space constraints or color figure charges

- Immediate publication on acceptance

- Inclusion in PubMed, CAS, Scopus and Google Scholar

- Research which is freely available for redistribution 